\title{
Waiting-time solutions of a nonlinear diffusion equation: Experimental study of a creeping flow near a waiting front
}

\author{
B. M. Marino, L. P. Thomas, R. Gratton, J. A. Diez, and S. Betelú \\ Instituto de Fisica Arroyo Seco, Facultad de Ciencias Exactas, \\ Universidad Nacional del Centro de la Provincia de Buenos Aires, Pinto 399, 7000 Tandil, Argentina
}

\section{J. Gratton}

Instituto Fisica del Plasma, Consejo Nacional de Investigaciones Cientificas y Técnicas, Facultad de Ciencias Exactas y Naturales, Universidad de Buenos Aires, Pabellón I, Ciudad Universitaria, 1428 Buenos Aires, Argentina

(Received 14 February 1996)

\begin{abstract}
We investigate an unsteady plane viscous gravity current of silicone oil on a horizontal glass substrate. Within the lubrication approximation with gravity as the dominant force, this current is described by the nonlinear diffusion equation $\phi_{t}=\left(\phi^{m} \phi_{x}\right)_{x}$ ( $\phi$ is proportional to the liquid thickness $h$ and $m=3>0$ ), which is of interest in many other physical processes. The solutions of this equation display a fine example of the competition between diffusive smoothening and nonlinear steepening. This work concerns the so-called waiting-time solutions, whose distinctive character is the presence of an interface or front, separating regions with $h \neq 0$ and $h=0$, that remains motionless for a finite time interval $t_{w}$ meanwhile a redistribution of $h$ takes place behind the interface. We start the experiments from an initial wedge-shape configuration $\left[h(x) \approx \alpha^{\prime}\left(x_{0}-x\right)\right]$ with a small angle $\left(\alpha^{\prime} \leqslant 0.12 \mathrm{rad}\right)$. In this situation, the tip of the wedge, situated at $x_{0}$ from the rear wall $\left(15 \mathrm{~cm} \leqslant x_{0} \leqslant 75 \mathrm{~cm}\right)$, waits at least several seconds before moving. During this waiting stage, a region characterized by a strong variation of the free surface slope (corner layer) develops and propagates toward the front while it gradually narrows and $\partial^{2} h / \partial x^{2}$ peaks. The stage ends when the corner layer overtakes the front. At this point, the liquid begins to spread over the uncovered substrate. We measure the slope of the free surface in a range $\approx 10 \mathrm{~cm}$ around $x_{0}$, and, by integration, we determine the fluid thickness $h(x)$ there. We find that the flow tends to a self-similar behavior when the corner layer position tends to $x_{0}$; however, near the end of the waiting stage, it is perturbed by capillarity. Even if some significant effects are not included in the above equation, the main properties of its solutions are well displayed in the experiments [S1063-651X(96)13309-2]
\end{abstract}

PACS number(s): 47.15.Gf, 47.10.+g, 68.10.Cr, 68.45.Gd

\section{INTRODUCTION}

We consider a nonlinear diffusion equation in plane symmetry of the form

$$
\frac{\partial \phi}{\partial t}=\frac{\partial}{\partial x}\left(\phi^{m} \frac{\partial \phi}{\partial x}\right), \quad m>0 .
$$

Equations of this type describe many interesting physical processes such as heat transport by radiation in partially or totally ionized gases, or by electrons in plasmas [1], unconfined ground-water flow and gas percolation through porous media [2], electric transmission in cables with resistive coatings, as well as other phenomena [3,4]. In particular, Eq. (1) with $m=3$ is obtained in the lubrication theory approximation [5], and describes viscous liquids flows over smooth horizontal substrates, also called creeping flows [5-7], which receive attention because of their environmental interest [8-11]. Since creeping flows can be studied by means of simple and cheap experiments, they are well suited to test some solutions of Eq. (1) in the laboratory, and to investigate some of their typical features $[6,12]$.

Let us note that by setting $\eta=\phi^{m}$ and $\tau=t / m$, Eq. (1) can be transformed into

$$
\frac{\partial \eta}{\partial \tau}=\left(\frac{\partial \eta}{\partial x}\right)^{2}+m \eta \frac{\partial^{2} \eta}{\partial x^{2}}
$$

which displays clearly that the time evolution of $\eta$ results from a combination of nonlinear wave propagation (first term on the right) and diffusion (second term) $[13,14]$.

Because of the nonlinearity of the diffusion term of Eq. (1a), there are solutions with well-defined fronts or interfaces $[15,16]$, separating regions where $\phi \neq 0$ and $\phi=0$. Fronts of this type are currently observed in creeping flows, where it is easy to measure with accuracy their propagation $[10,17]$ and their cross profile [12]. There is also much evidence of their existence in the other phenomena described by Eq. (1), much harder to study experimentally $[18,19]$. An interesting property is displayed by the waiting-time solutions [4,13,20-24] which describe an evolution of $\phi(x)$ behind a front at a fixed position during a finite waiting time $t_{w}$. The waiting-time solutions have aroused a considerable interest among the mathematicians, but the related phenomenology has not yet been studied experimentally in a systematical way. Since in the literature there are few references of observations, mostly qualitative, of waiting-time creeping flows [4,25], we believe it is of interest to report the results of a series of experiments specifically addressed to investigate a viscous current near a waiting front. 
Now we briefly recall some properties of waiting-time solutions to provide a theoretical background. First we mention that the exact evaluation of $t_{w}$ for given initial conditions is still an open problem. Formulas for lower and upper bounds of $t_{w}$ are known $[4,13,14,26,27]$. The bounds given by Aronson and co-workers [14,26] and Vazquez [27] are particularly relevant to our concern.

In a noteworthy paper, Kath and Cohen [13] considered the limit $m \ll 1$, in which the leading term of Eq. (1a) corresponds to a nonlinear first order wave propagation equation. The solutions, solved by the method of characteristics, may develop discontinuities of the first derivative $\partial \phi / \partial x$ (corner shocks). The diffusion term included in Eq. (1a) smooths the changes of $\partial \phi / \partial x$; therefore, a corner shock becomes a corner layer, i.e., a small $x$ interval in which $\partial^{2} \phi / \partial x^{2}$ has a peak. By using this analysis Kath and Cohen discussed the evolution of initial profiles $\phi \propto\left|x-x_{0}\right|^{p}$ near the front $\left(x_{0}\right.$ is the front position). Then the formation of a corner shock determines the basic behavior of the waiting-time solutions. The front can start to move depending upon whether the corner shock forms just at, or behind the front. They found the following.

(a) If $0<p<2 / m$, the front moves immediately ( $t_{w}=0$ ).

(b) If $p=2 / m$, the front begins to move after a finite waiting time, and precisely then a corner shock is formed at $x_{0}$; this is the only case in which there is a formula $[13,14,26]$ for $t_{w}$ (that also holds for finite $m$ ).

(c) If $p>2 / m$, the front remains at rest for a finite time; at a certain moment during this interval a corner shock appears behind the front, and propagates overtaking the front at $t=t_{w}$.

The existence of similarity solutions with waiting time was proved by Lacey, Ockendon, and Tayler [4] using a phase-plane formalism. They found similarity solutions of the second kind, valid before and after $t_{w}$. They classified the solutions according to the self-similarity exponent $\delta(\delta>1$ in a continuous spectrum), and gave prescriptions for their construction, but did not discuss their properties. This type of solution may describe the asymptotic solutions near the front and close to $t_{w}$ of an initial value problem that displays a waiting-time behavior. The value of $\delta$ depends on the initial conditions of the problem, and its determination was not addressed by these authors. More recently, Gratton and Vigo [24] studied in detail the solutions of Lacey, Ockendon, and Taylor (their study focused on the case $m=3$, but the generalization to any $m$ is straightforward) and found that those solutions corresponding to $1<\delta<\frac{13}{10}$ display corner layers during the waiting time. They also numerically solved the initial value problem for profiles of the form $\left|x-x_{0}\right|^{p}$. They obtained a nonvanishing $t_{w}$ only for $p>\frac{2}{3}$, with $1<\delta<\frac{13}{10}$, and observed always the formation of a corner layer behind the front [as in case (c) above] except for $p=$ $\frac{2}{3}$, when a corner shock appears at $t=t_{w}$ and $x=x_{0}$; for $p<\frac{2}{3}$ the front moves at once $\left(t_{w}=0\right)$. Thus the numerical solutions have extended the validity of the results of Kath and Cohen [13] beyond the condition $m \ll 1$.

The way in which the front begins to move also depends on $p$. So, if the waiting time is due to purely local effects, the interface begins to move smoothly [26], i.e., the front velocity does not jump at $t=t_{w}$. Conversely, if nonlocal effects are dominant, the front starts to move with a finite velocity.
In this work we describe systematic experimental studies of situations dominated by the waiting front phenomenology. Specifically, we are concerned with viscous gravity currents under the conditions that allow us to use the lubrication approximation. Then the appropriate equation of motion is Eq. (1) with $m=3$, and the diffusing scalar is $\phi=(g / 3 \nu)^{1 / 3} h$, with $g$ the gravity and $\nu$ the kinematics viscosity. In the initial configuration the fluid has a wedgelike shape with an aspect ratio $\alpha=h_{0} / x_{0}$ ( $h_{0}$ is the wedge height). This shape is easily prepared by keeping the fluid at rest for a convenient time inside an inclined rectangular tray, which is then quickly brought to a horizontal position at $t=0$. As initially $h=\alpha\left(x_{0}-x\right)$, and $p=1>\frac{2}{3}$, the flow has a waiting front and a corner layer is formed for $t<t_{w}$. For this case, the most restrictive bounds for $t_{w}$ are given by Vazquez [27] (see also [26]): $0.52<t_{w} / t^{*}<29.87$, where $t^{*}=(3 \nu / g)\left(x_{0}{ }^{2} / h_{0}{ }^{3}\right)$ is a characteristic time that results from the nondimensional form of Eq. (1).

We carry out a series of experiments with $x_{0} \approx 15,50$, and $75 \mathrm{~cm}, 0.012 \leqslant \alpha \leqslant 0.12$ and fluids with viscosity $\nu \approx 7,10$, 20 , and $100 \mathrm{~cm}^{2} \mathrm{~s}^{-1}$. In all the experiments the front moves only after a long time $\left(10-10^{3} \mathrm{~s}\right.$, depending on $\alpha, x_{0}$ and $\nu$ ), with $t_{w} / t^{*}$ well in the above range. A corner layer forms before $t_{w}$, as is evident in the $\partial h / \partial x$ distribution, which develops a peak that grows and narrows as it moves toward the front. The peak becomes increasingly asymmetrical, with a very steep leading border, followed by a broader rear region. The position and height of the peak tend to follow power laws of $\left(t_{w}-t\right)$, which suggests the approach to a self-similar regime. However, when the corner layer comes very close to $x_{0}$, the power law behavior for the amplitude of the $\partial h / \partial x$ peak breaks down. The leading border of the peak overtakes the front; then the waiting stage ends and the liquid spreads beyond $x_{0}$. The values of $\delta$ observed are very close to the unity, thus suggesting that the front starts to move with nonzero velocity.

We address considerable work to study whether the laboratory flows are properly described by Eq. (1). The main limitations are due to effects caused by surface forces and departures from the geometry of the velocity field at the basis of the lubrication approximation. They will be considered in Sec. II, and discussed when used for the interpretation of the results. We also provide numerical solutions of Eq. (1) with or without the inclusion of a term accounting for the Laplace pressure. These solutions are obtained through an adaptation of the Crank-Nicholson method employed by Diez et al. [28].

In spite of these limitations, the simple flow studied in this work clearly displays much of the typical phenomenology related to the mathematical properties of Eq. (1). The most noticeable feature is the behavior of the $\partial h / \partial x$ peak, whose formation and evolution are deeply associated with the combination of a nonlinear wave propagation and a diffusion that is typical of nonlinear diffusion. We believe that the experimental results reported here are good to orient theoretical formulations and to validate numerical simulations.

\section{DESCRIPTION OF THE EXPERIMENTS}

\section{A. Experimental setup}

We use a rectangular glass tray (width $0.72 \mathrm{~m}$, length 0.98 $\mathrm{m}$, height $0.10 \mathrm{~m}$ ), which can rotate about a horizontal axis at 
$38 \mathrm{~cm}$ from the rear end. The large width of the tray ensures that the sidewalls do not affect the flow in the central region; then the magnitudes are independent of the transverse coordinate. After carefully cleaning the spreading surface with hexane, we introduce the liquid into the tray, previously tilted by the desired angle respect to the horizontal. Capillary effects tend to distort the wedge near the front; we reduce this effect by starting the experiment at the moment when the fluid has a well defined wedgelike shape. Several hours or even days may be required to achieve a good initial profile, depending on the fluid viscosity and on the values of $\alpha$ and $x_{0}$. To start the experiment, we let the tray fall to the horizontal position (determined by stops). We use a dashpot to reduce the vibrations due to the impact of the heavy (weight $\approx 25 \mathrm{~kg}$ ) glass-fluid system against the stops. We measure the horizontality of the spreading surface and the aspect ratio $\alpha$ with an error less than $10^{-4}$ by means of the deviation of a laser beam reflected by the tray surface. The flatness departures with respect to a horizontal average ideal plane are less than $3 \times 10^{-2} \mathrm{~cm}$. In this way, at $t=0$, $h(x)=\alpha\left(x_{0}-x\right)$.

\section{B. Conditions on the experimental parameters}

The reported data correspond to 35 experiments which cover a wide range of $\nu, \alpha$, and $x_{0}$. The choice of these parameters is limited because of the approximation needed to derive Eq. (1) from the Navier-Stokes equation. The width of the tray is at least $\approx x_{0}$, since the side walls may affect the plane geometry. We observe the central region of the spreading in order to detect eventual transverse dependencies of the front position, thus indicating departures from plane geometry. According to these observations, we can safely affirm that our results are not significantly affected by the lateral walls. The inertial term may be neglected if $\alpha R \ll 1$ [29], where $R \cong U h_{0} / \nu$ is the Reynolds number, with $U=x_{0} / t^{*}$ the characteristic velocity (we pose $R \leqslant 0.02$ ). In addition, $t^{*}$ must be larger than the time of fall of the tray, of the order of some tenths of a second (we pose $t^{*} \geqslant 10 \mathrm{~s}$ ). On the other hand, the lubrication approximation requires that $(\partial h / \partial x)^{2} \ll 1$ everywhere. Initially, this condition is satisfied if $\alpha^{2} \ll 1$ [29] (we pose $\alpha \leqslant 0.12$ ). However, as the flow evolves, $\partial h / \partial x$ may become much larger than $\alpha$ in some regions, so that the above condition does not ensure the smallness of $\partial h / \partial x$ there. Moreover, near the rear wall of the tray, the adherence of the fluid to the wall may affect strongly the flow. The possible influence of these local departures from the lubrication approximation on the measured magnitudes will be discussed in Sec. II C. The conditions on $R$ and $t^{*}$ are fulfilled by employing polydimethylsiloxanes (PDMS's) of high viscosity ( $\nu \cong 7,10,20$, and $100 \mathrm{~cm}^{2}$ $\mathrm{s}^{-1}, \rho=0.975 \mathrm{~g} \mathrm{~cm}^{-3}$ ), known to behave as Newtonian liquids for $\nu \leqslant 10 \mathrm{~cm}^{2} \mathrm{~s}^{-1}$, and for larger $\nu$ within the shear rates of the experiments $\left(U / h_{0} \leqslant 1 \mathrm{~s}^{-1}\right)[30,31]$. As we shall see in Sec. III, flows with equal $x_{0}$ and $\alpha$ but different $\nu$ give the same results, thus confirming that departures of the Newtonian behavior are negligible.

The influence of surface forces is of particular interest. During the waiting stage, they enter through the Laplace pressure generated by the surface tension $\gamma$ at the liquid-air interface $\left(\gamma=21.6 \mathrm{dyn} \mathrm{cm}^{-1}\right.$ for PDMS). By assuming $\partial h / \partial x \ll 1$ consistently with the lubrication approximation, the term

$$
-\frac{\gamma}{3 \rho \nu} \frac{\partial}{\partial x}\left(h^{3} \frac{\partial^{3} h}{\partial x^{3}}\right)
$$

should be added to the right hand of Eq. (1) ( $\rho$ is the liquid density). By introducing the capillary length $a=(\gamma / \rho g)^{1 / 2}(\approx 0.15 \mathrm{~cm}$ for PDMS $)$, the ratio between the term (2) and the right hand of Eq. (1) is given by

$$
a^{2}\left(\frac{\partial^{3} h}{\partial x^{3}} / \frac{\partial h}{\partial x}\right) \approx \frac{a^{2}}{(\Delta x)^{2}},
$$

where $\Delta x$ is the length scale of a slope variation (in our case, the width of the corner layer). Thus surface tension is important when this scale is of the order or smaller than $a$, irrespectively of the height scale. On the other hand, the width of the corner layer should scale as $x_{0}$. Therefore, the larger $x_{0}$, the smaller the portion of the current where the Laplace pressure may affect the corner layer and, therefore, the smaller its effect on the bulk. Note that $\alpha$ does not enter the problem.

After $t_{w}$ the situation changes, since we deal with a spreading over an initially uncovered substrate. Equation (1) must now be solved with a boundary condition appropriated for a moving contact line. According to usual approaches $[32,33]$ and considering that PDMS completely wets glass, this introduces the capillary number $\mathrm{Ca}=U /(\gamma / \rho \nu)=\alpha^{3}\left(x_{0} / a\right)^{2} / 3$ into the problem; specifically, Ca should be much larger than unity to make surface effects negligible. As we ought to work with small $\alpha$, this condition is not properly fulfilled in many experiments. Note that now $\alpha$ enter the problem even within the lubrication approximation.

We notice that capillarity may also produce a displacement $\Delta x$ of the front in the time $0<t<t_{w}$. Its magnitude can be estimated through the well known Tanner's law $[28,32,33] \theta_{\alpha} \cong 4.2 \mathrm{Ca}^{1 / 3}$ which relates the apparent contact angle $\theta_{\alpha}$ with the advancing front velocity $U$. By setting $\Delta x=U t_{w}, \theta_{\alpha}=\alpha$, the requirement $\Delta x \ll x_{0}$ yields

$$
0.4 \frac{a^{2}}{x_{0}^{2}} \ll 1
$$

This condition is amply satisfied in our experiments.

\section{Measurement techniques}

As PDMS's are very transparent liquids, it is convenient to observe the free surface through light refraction (refraction index $\eta \cong 1.40$, with a slight dependence on the viscosity). We measure the free surface slope by means of the deviation of a light slice composed of vertical parallel rays, which forms an angle of $45^{\circ}$ with the flow direction ( $x$ axis) (Fig. 1). The light slice $a$ (see the inset in Fig. 1) enters the fluid from below in such a way that part of it ( $b$ ) goes through the substrate only, and therefore maintains the vertical direction, while the remaining part $(c)$ is refracted at the liquid free surface. The rays of part $c$ are deviated only in the $x$ direction by amounts that depend on the local surface slope. Parts $b$ and $c$ produce a bright curve composed by two pieces $b^{\prime}$ 


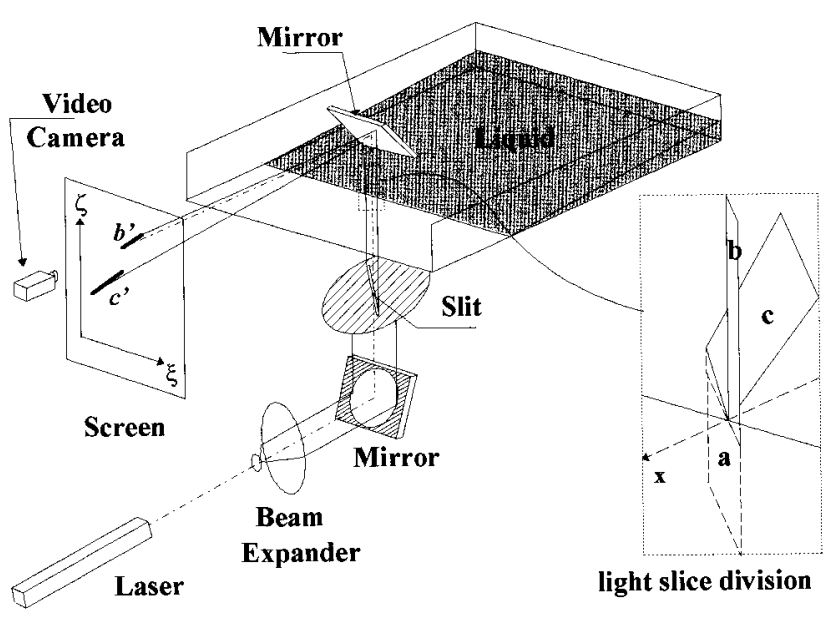

FIG. 1. Experimental setup to measure the $\partial h / \partial x$ profile.

and $c^{\prime}$ on a semitransparent screen and are recorded by means of a video camera. The $(\xi, \zeta)$ coordinates on the screen corresponding to a point of the curve are related to the $x$ coordinate of the incident ray and the slope of the free surface at $x$, respectively. By supposing that the distance $D$ between the fluid surface and the screen is much greater than $h$, and with a convenient choice of the origin, these coordinates are

$$
\begin{gathered}
\xi=\zeta=x \text { for } b^{\prime} ; \\
\xi=x \text { and } \zeta=x+D \operatorname{tg} \theta \text { for } c^{\prime},
\end{gathered}
$$

where the angular deviation $\theta$ is related with the slope $\partial h / \partial x$ by

$$
\frac{\partial h}{\partial x}=\frac{\sin \theta}{\eta-\cos \theta} \approx \frac{\theta}{\eta-1} \text { for } \theta \ll 1 .
$$

Initially, $b^{\prime}$ and $c^{\prime}$ are parallel straight segments (as we show in Fig. 1), because the liquid free surface has a uniform slope (note that immediately after the fall of the tray, the method gives a measurement of $\alpha$ which must be consistent with the value obtained from the laser beam reflection). As the flow develops, segment $c^{\prime}$ distorts, portraying the changes of the slope of the free surface. The range of observable slopes is limited in theory by the internal total reflection in the liquid-air surface (that sets a bound of $\partial h / \partial x \cong 0.8$ for PDMS). In practice, the maximum deviation of the rays collected by our setup is $\theta \approx 0.3 \mathrm{rad}(\partial h / \partial x \approx 0.67)$. After image processing, the indeterminacy of the measurements is less than $2.10^{-3}$ for $\partial h / \partial x$ and $0.25 \mathrm{~mm}$ for the $x$ coordinate.

As a complement to the previous technique we record images of the zone near the front by means of a zoom $\times 100$ video camera from a lateral position slightly above the spreading surface. To keep the front region centered in the video screen, the camera is manually displaced along a rail (75-cm length) parallel to the flow direction. By interposing a partially reflecting surface, we form the virtual image of a fixed reference scale, which therefore appears superimposed on the current image. The current is illuminated by a diffuse light source placed at the side of the tray opposite to the camera, slightly below the surface. The position $x_{c}$ of the leading border of the corner layer is given by the foremost edge of a dark band due to the strong deviation of the rays traversing the large slope region (note that, near $t_{w}, x_{c}$ is very close to the coordinate $x_{m}$ of the point of maximum slope). The technique gives also $x_{0}$, the waiting time $t_{w}$, and the position $x_{f}$ of the most advanced part of the moving front after $t_{w}$. The positions are measured within $\approx 0.1 \mathrm{~mm}$, i.e., with precision higher than in the light slice method. Since part of the information $\left(t_{w}, x_{c}\right.$, and $\left.x_{f}\right)$ is given by both techniques, the consistency of the results strengthens their reliability.

\section{RESULTS}

\section{A. Global aspects. Waiting-time measurement}

In all the experiments there is a waiting stage, during which the front remains at rest within the accuracy of the detection systems $\left(\approx 10^{-3} x_{0}\right)$. In this stage, the flow always displays the same qualitative behavior. The initial triangular profile becomes increasingly curved, and two distinct regions become recognizable. In a first region, extending from the rear wall $(x=0)$ to a certain intermediate position $x_{i}\left(<x_{0}\right)$, the profile strongly departs from the initial condition; except near the rear wall, the slope increases monotonically from almost zero (for $x \approx 0$ ) to values considerably steeper than $\alpha$ at $x_{i}$. This region is followed by another which extends from $x_{i}$ up to $x_{0}$; in this domain the initial profile remains practically unchanged. The transition between these regions moves toward $x_{0}$ and becomes progressively sharper, gradually acquiring the characteristics of a corner layer. Figure 2 shows the evolution of $\partial h / \partial x$ and $h$ distributions (the last one is obtained by integrating the first curves) vs $\left(x-x_{0}\right)$ for a typical current. The slope profiles of Fig. 2(a) indicate that as the corner layer advances it narrows and the maximum slope increases. The peak of $\partial h / \partial x$ becomes increasingly asymmetric as the extreme of $\partial^{2} h / \partial x^{2}$ shifts forward. Looking at Fig. 2, one notices that the distribution of $\partial h / \partial x$ ahead to the corner layer remains practically unchanged. As a result the flow closely resembles a spreading over a fixed wedge profile. However, sensitive measurements based on the reflection of a laser beam show that the slope in the foremost region varies slightly, but the changes are too small to be seen in Fig. 2. The waiting stage ends when the corner layer overtakes the front, which then starts to move, thus defining the waiting time $t_{w}$.

In Fig. 3, we show $T_{w}=t_{w} / t^{*}$ as a function of $\alpha$ for different values of $x_{0}$ and $\nu$. The dispersion is because small errors of the $\alpha$ measurements are magnified by the cubic dependence of $t^{*}$ on $\alpha$, and because the viscosity is measured within $2 \%$ accuracy by standard techniques (the value of $\nu$ is modified according to the fluid temperature). The lubrication theory approximation predicts a unique value for $T_{w}$; however, we observe that $T_{w}$ depends slightly (roughly linearly) on $\alpha$. The extrapolated value for $\alpha \rightarrow 0$ is close to the value (0.963) obtained by numerically solving Eq. (1) (with $\gamma=0$ ). Note that they are within the bounds given by theoretical predictions [14,26,27].

We ascribe the dependence of $T_{w}$ on $\alpha$ to a twodimensional effect coming from the rear wall of the tray, which imposes a vertical no slip boundary condition that 


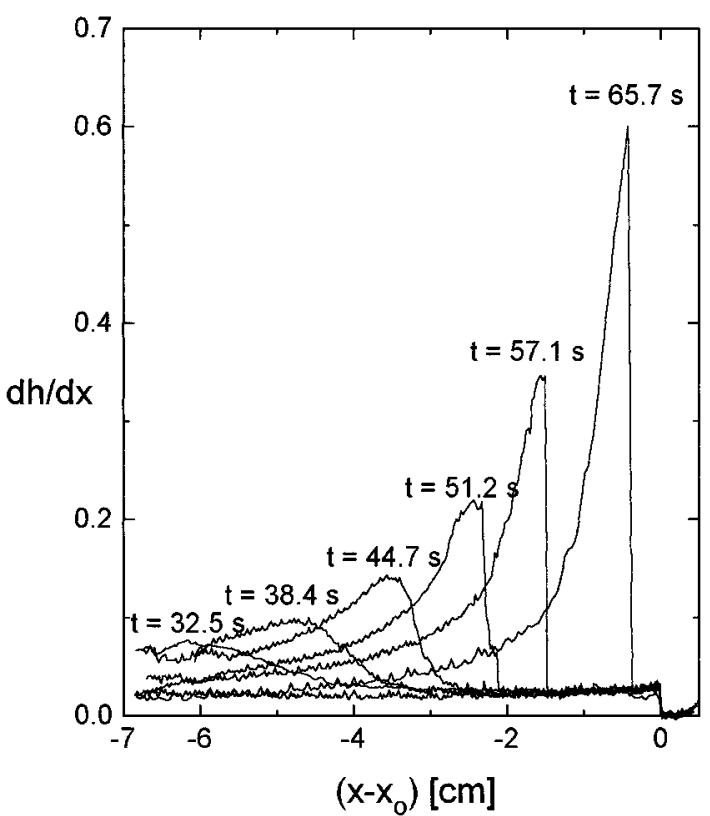

(a)

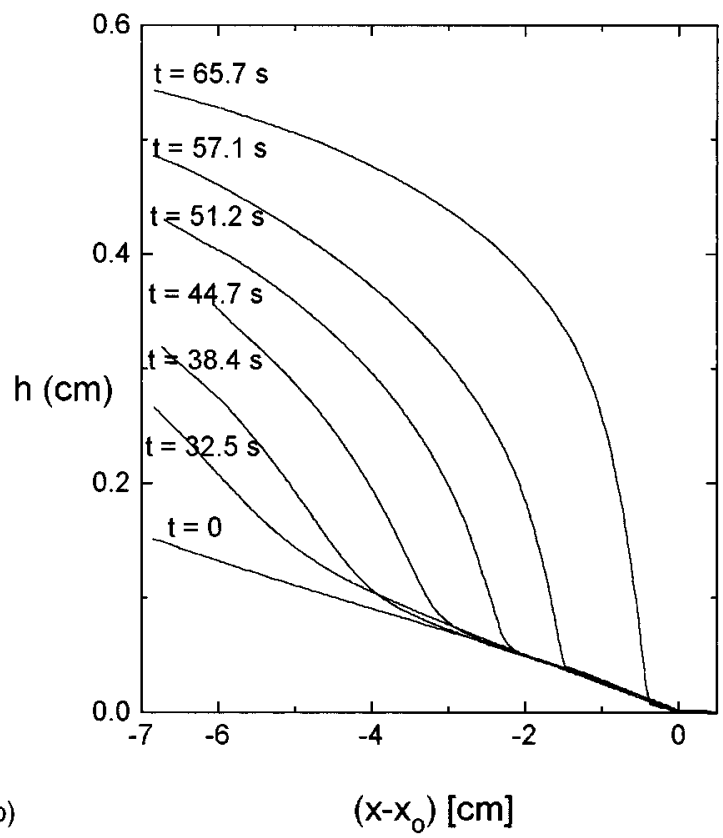

FIG. 2. Sequences of (a) slope profiles and (b) height profiles for a typical experiment $\left(\alpha=0.0229, x_{0}=78 \mathrm{~cm}\right.$, and $\nu=22.7$ $\left.\mathrm{cm}^{2} \mathrm{~s}^{-1}\right)$.

cannot be properly treated within the lubrication approximation. Then the solution of Eq. (1) does not correctly describe the flow near the rear wall. A heuristic estimate of this effect can be made by assuming that a slab of fluid of width proportional to $h_{0}$ adjacent to the rear wall simply does not participate in the spreading. Hence $x_{0}$ should be replaced by an effective length $\left(x_{0}-c h_{0}\right)$, where $c$ is a constant of the order of unity. This leads to a corrected characteristic time

$$
t^{*} \cong \frac{3 \nu}{g \alpha^{3} x_{0}}(1+c \alpha)
$$

Therefore $T_{w} \propto(1+c \alpha)$, according to the linear dependence of Fig. 3. The best fit line gives $c \approx 2$.

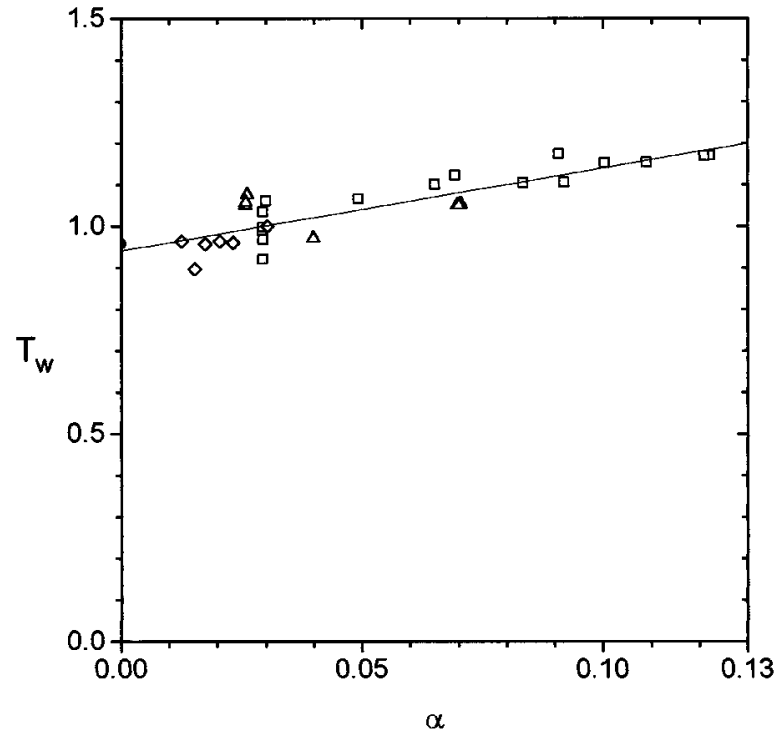

FIG. 3. Dimensionless waiting time as function of the initial aspect ratio for $\square, x_{0} \approx 15 \mathrm{~cm} ; \Delta, x_{0} \approx 50 \mathrm{~cm} ; \diamond, x_{0} \approx 75 \mathrm{~cm} ; \boldsymbol{\bullet}$, numerical value (obtained with a one-dimensional code) for $\gamma=0$. The best linear fit is $T_{w}=0.94+1.99 \alpha$.

We have also examined other possible causes of the $T_{w}(\alpha)$ dependency, but they should all be rejected. First, the variation is too large to be explained by eventual departures from the lubrication approximation in the bulk of the current. In fact, $\partial h / \partial x$ is small everywhere except during a time interval much shorter than $t_{w}$ and within a region of size $\ll x_{0}$. Second, the influence of the capillarity term given by (2) on Eq. (1) must be ruled out because $T_{w}$ does not depend on $x_{0}$. Finally, the data shown in Fig. 3 correspond to experiments with various liquids covering a wide range of viscosity. Therefore, the observed variation of $T_{w}$ cannot be ascribed to non-Newtonian effects.

As a consequence, from now on we shall use the effective length $\left(x_{0}-c h_{0}\right)$ instead of $x_{0}$. This change, by definition, yields a modified $T_{w}$ that no longer depends on $\alpha$. It also cancels small dependencies on $\alpha$ in other experimental variables thus enlightening the discussions.

\section{B. Evolution of the corner layer and the moving front}

In Fig. 4(a) we show $X_{C}=\left(x_{C}-x_{0}\right) / x_{0}$ and $X_{f}=\left(x_{f}-x_{0}\right) / x_{0}$ as functions of $T=\left(t-t_{w}\right) / t_{w}$ for different values of $\alpha$ and $x_{0} \cong 15 \mathrm{~cm}$. Similar results are also obtained for $x_{0} \cong 50$ and $75 \mathrm{~cm}$. Curves $X_{C}(T)$ and $X_{f}(T)$ join smoothly across $T=0$, so that the front motion appears to be a natural continuation of the corner layer motion. However, all the curves $X_{C}(t)$ are almost coincident, while the curves $X_{f}(T)$ differ slightly, opening like a fan. The data for $-0.05<T<0$ are well approximated by a power law with an exponent $\delta_{b}=1.13$ [see Fig. 4(b)], close to the value (1.07) obtained in the numerical solution of Eq. (1) without surface tension. This suggests that the flow near $x_{0}$ tends to selfsimilarity for $t \rightarrow t_{w}$ independently on $\alpha$. For $0<T<0.05$ the curves may also be approximated by power laws with exponents $\delta_{a}$ close to unity (the numeric value is 0.995 ), which 


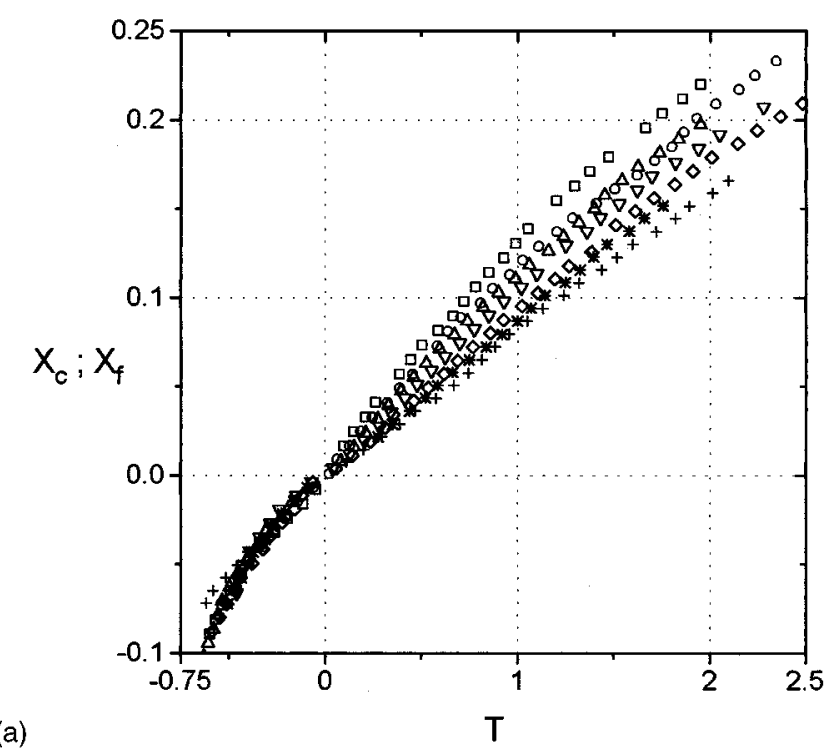

(a)

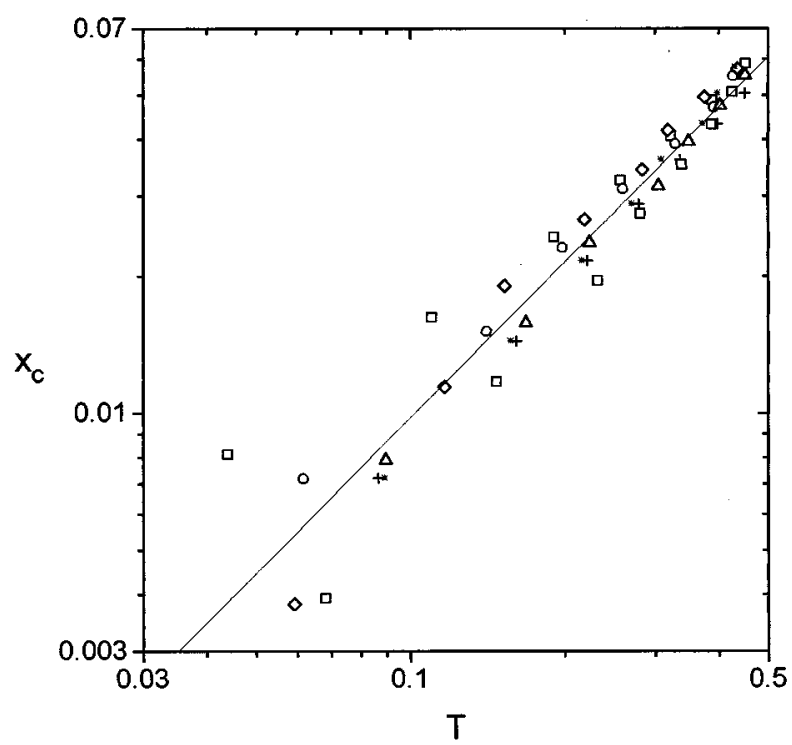

FIG. 4. (a) Dimensionless coordinate of the corner layer $X_{C}(T)$ and of the front $X_{f}(T)$ in the cases $x_{0} \cong 15 \mathrm{~cm} \mathrm{( \square ,}$ $\alpha=0.12 ; \bigcirc, \alpha=0.10 ; \Delta, \alpha=0.09 ; \nabla, \alpha=0.08 ; \diamond, \alpha=0.065$; *, $\alpha=0.05$; and,$+ \alpha=0.03$ ). Observe the coincidence among the experimental results for $T<0$ and the fan-shaped disposition of the points sequences for $T>0$. (b) Log-log representation of the same cases of (a) for $T>-0.5$.

agrees with mathematical predictions [26,27]. Nevertheless, the corresponding prefactors increase while $\delta_{a}$ slowly decreases with $\alpha$.

When we compare the results for various $x_{0}$ and fixed $\alpha$ (see Fig. 5), as before we observe that the $X_{C}(T)$ curves coincide while the $X_{f}(T)$ curves differ slightly. $X_{C}(T)$ is again well approximated by a unique power law with an exponent $\delta_{b}$ close to that obtained for the curves of Fig. 4(b), while the $X_{f}(T)$ curves are fitted by power laws with the exponents close to unity and prefactors increasing with $x_{0}$.

These results indicate that, within the parameter range covered by our measurements, the motion of the corner layer is practically independent of $\alpha$ and $x_{0}$; therefore capillarity does not significantly affect this motion. This agrees with the

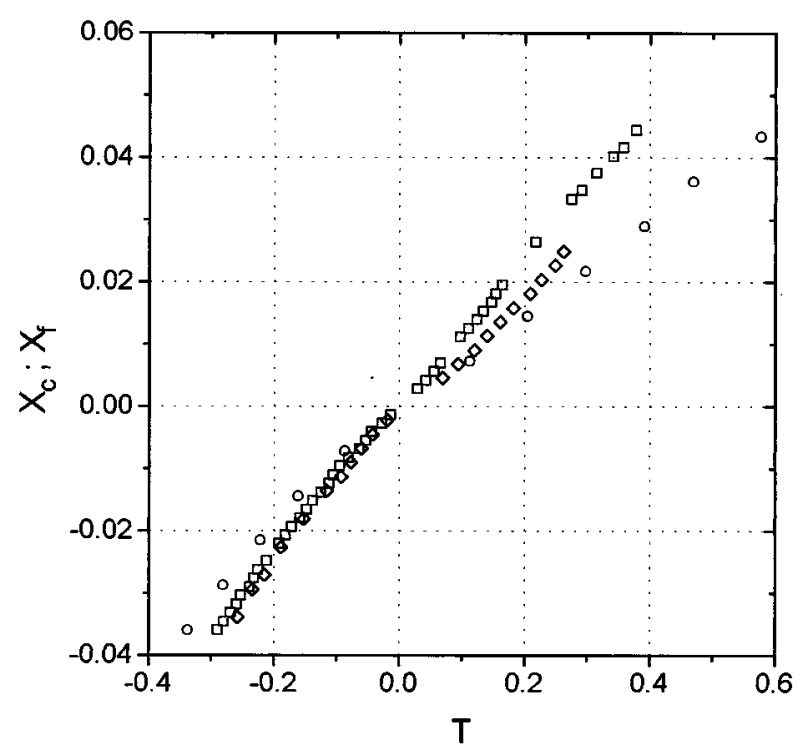

FIG. 5. Dimensionless coordinate of the corner layer $X_{C}(T)$ and of the front $X_{f}(T)$ in the cases $\alpha \approx 0.03\left(\bigcirc, x_{0}=15 \mathrm{~cm} ; \diamond\right.$, $x_{0}=50 \mathrm{~cm}$; and $\left.\square, x_{0}=75 \mathrm{~cm}\right)$. Note the fan-shaped disposition of the points for $T>0$.

numerical solutions of Eq. (1) including the capillarity term (2). We point out that the comparison between $X_{C}(T)$ and the numerical solution is meaningful because the maximum slope is always small $(<0.6)$ for all values of $X_{C}$ considered.

On the other hand, the experimental curves $X_{f}(T)$ depend on an unseparable combination of $\alpha$ and $x_{0}$, as expected since the capillary number $\mathrm{Ca}$ becomes the relevant parameter. As we see in Fig. 6, the data of cases corresponding to the same Ca but different $x_{0}$ and $\alpha$ fall very close to a unique line. This means that the fanning out of the $X_{f}(T)$ curves is due to the influence of capillarity on the motion of the spreading front. This effect should decrease as $\mathrm{Ca}$ increases;

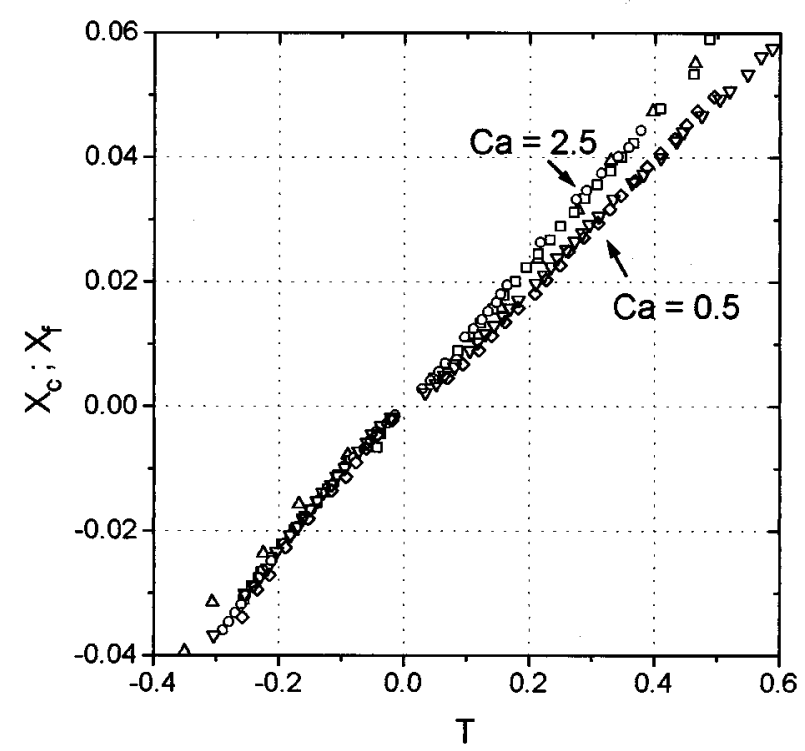

FIG. 6. Dimensionless coordinate of the corner layer $X_{C}(T)$ and of the front $X_{f}(T)$ in cases labeled according to the value of $\mathrm{Ca}$. Note the coincidence of $X_{f}(T)$ in the cases with the same Ca. 


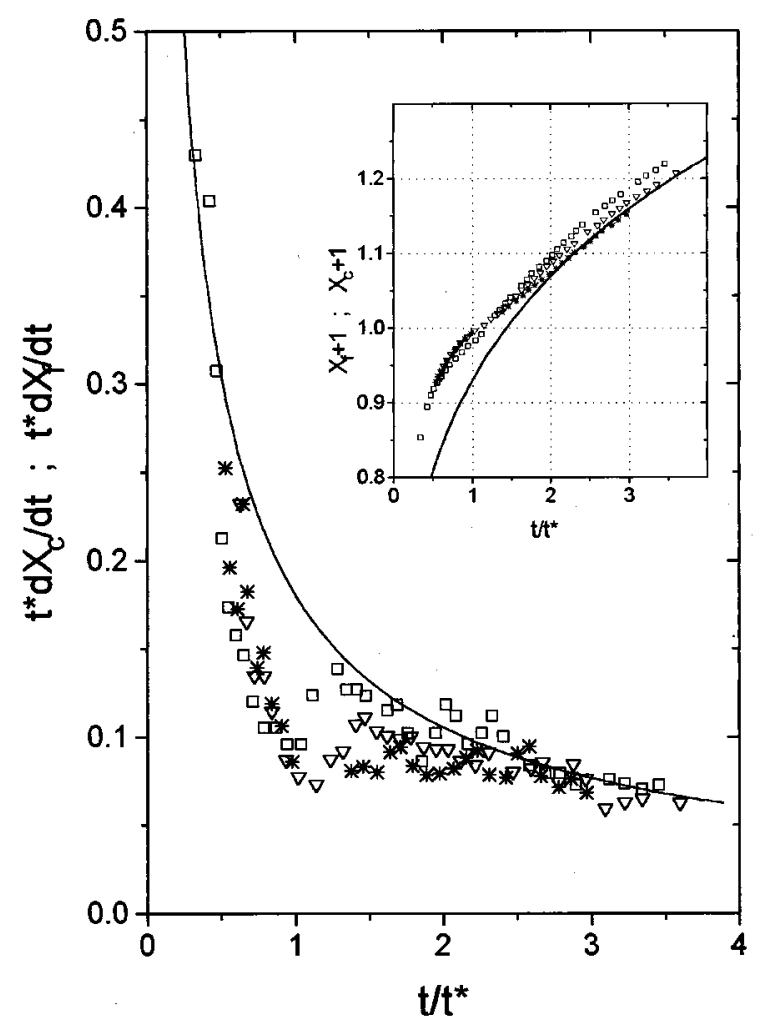

FIG. 7. Dimensionless velocity of the corner layer and the front obtained by derivation for the three cases reported in Fig. 4 (see the inset). The solid curve corresponds to the analytical self-similar solution given by Eq. (3) and the symbols to $\square, \mathrm{Ca}=5.76 ; \nabla$, $\mathrm{Ca}=1.71$; and $\star, \mathrm{Ca}=0.42$.

we observe that all the best fit power laws practically coincide for $\mathrm{Ca}>4$.

\section{Comparison with an analytical self-similar regime}

It is noteworthy to compare the corner layer and the front evolution with the analytical self-similar solution (of the first kind) of Eq. (1) corresponding to the fluid concentrated at the origin at $t=0[6,12,15]$. This solution is also used by Vazquez [27] to find an upper bound of $t_{w}$. Here, the front position is given by

$$
x_{f}=\lambda\left(\frac{g A^{3}}{3 \nu} t\right)^{\delta}
$$

where $\delta=0.2, \lambda=1.411 \ldots$, and $A$ is the area of the fluid lateral cross section. In the inset of Fig. 7 we report with adequate scales the positions of the corner layer and the moving front for three cases yet shown in Fig. 4(a) (symbols) with the front position given by Eq. (3) (full line). An important point is that the latter reaches $x_{0}$ at a time $t=1.43 t^{*}$, close to the experimental waiting time. From Fig. 7 , it is clear that the velocities of the experimental fronts are close to the velocity given by the time derivative of Eq. (3). In all the cases, we observe a decrease of the corner layer velocity for $t \leqslant t_{w}$ according to an exponent $\delta_{b}>1$. The front starts to move with a finite velocity (that is, $\delta_{a} \approx 1$ ), which corresponds to a sudden increase of velocity in Fig. 7 [as evident in the case $\mathrm{Ca}=5.76(\square)]$ by a sudden increase at

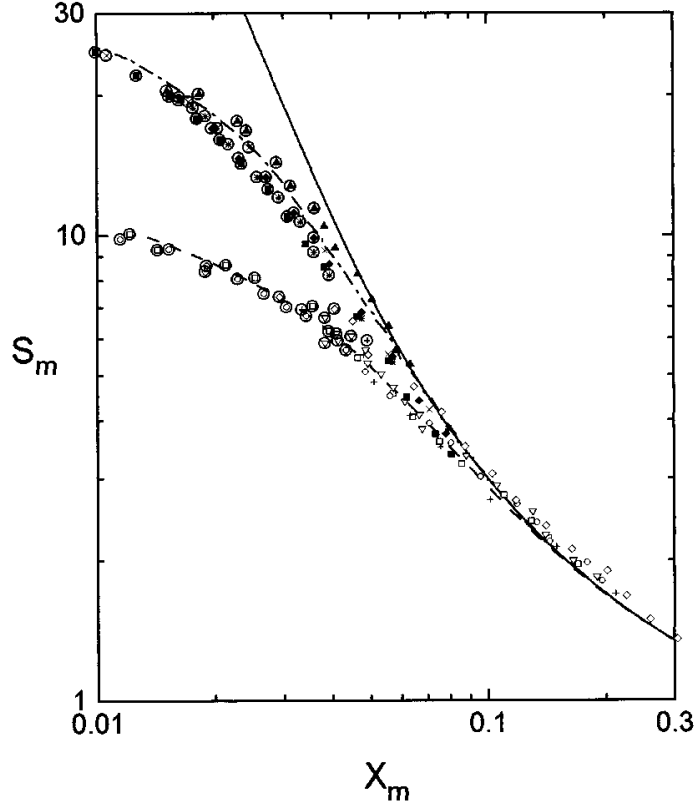

FIG. 8. Dimensionless maximum slope $S_{m}\left(X_{C}\right)=s_{m} / \alpha$ as a function of its coordinate $X_{m}$. The curves correspond to numerical values with $\gamma=0$ (solid line) and $\gamma=21 \mathrm{dyn} / \mathrm{cm}^{2}$ for $x_{0} \cong 15 \mathrm{~cm}$ (dashed line) and $75 \mathrm{~cm}$ (dash-dot line). The symbols correspond to the following cases: $\diamond, \alpha=0.065, x_{0}=15.3 \mathrm{~cm}$, and $\nu=100.5$ $\mathrm{cm}^{2} \mathrm{~s}^{-1} ;+, \alpha=0.0492, x_{0}=15.2 \mathrm{~cm}$, and $\nu=102.5 \mathrm{~cm}^{2} \mathrm{~s}^{-1}$; $\bigcirc, \quad \alpha=0.0304, x_{0}=13.85 \mathrm{~cm}, \quad$ and $\nu=101.2 \mathrm{~cm}^{2} \mathrm{~s}^{-1}$; $\nabla, \quad \alpha=0.0293, x_{0}=14.7 \mathrm{~cm}, \quad$ and $\nu=20.9 \mathrm{~cm}^{2} \mathrm{~s}^{-1}$; $\square, \quad \alpha=0.0295, x_{0}=14.8 \mathrm{~cm}, \quad$ and $\nu=8.98 \mathrm{~cm}^{2} \mathrm{~s}^{-1}$; $\star \quad \alpha=0.0233, x_{0}=77.0 \mathrm{~cm}, \quad$ and $\nu=20.9 \mathrm{~cm}^{2} \mathrm{~s}^{-1}$; $\checkmark, \quad \alpha=0.0205, x_{0}=76.5 \mathrm{~cm}, \quad$ and $\nu=21.6 \mathrm{~cm}^{2} \mathrm{~s}^{-1}$; 口, $\alpha=0.017, x_{0}=76.74 \mathrm{~cm}, \quad$ and $\nu=21.3 \mathrm{~cm}^{2} \mathrm{~s}^{-1}$; - $\alpha=0.0153, x_{0}=77.7 \mathrm{~cm}$, and $\nu=21.6 \mathrm{~cm}^{2} \mathrm{~s}^{-1} ;$ and $\Delta, \alpha=0.0126, x_{0}=75.5 \mathrm{~cm}$, and $\nu=7.49 \mathrm{~cm}^{2} \mathrm{~s}^{-1}$.

$t_{w}$; this increase becomes less evident as Ca decreases ( $\Delta$ and $\star)$. For $t \gg t_{w}$ and $x_{f} \gg x_{0}$ the position of the front agrees well with Eq. (3) irrespective of the initial conditions. As this asymptotic regime has been widely studied in previous work, we omit further details here.

\section{Evolution of the maximum slope}

We now discuss the evolution of the maximum slope $s_{m}$ of the current profile during the waiting stage. The technique described in Sec. II allows us to measure $s_{m}$ up to a time quite close to $t_{w}$ before $s_{m}$ becomes too large to be captured in the screen.

Figure 8 is a $\log -\log$ plot of the experimental points $S_{m}=s_{m} / \alpha$ vs $X_{m}=\left(x_{0}-x_{m}\right) / x_{0}$, for different values of $\alpha$ and $\nu$. We also show the results of numerical solutions including the capillary term $\left(\gamma=21.64 \mathrm{dyn} \mathrm{cm}^{-1}\right)$ for $x_{0}=15 \mathrm{~cm}$ (dashed line) and $75 \mathrm{~cm}$ (dash-dot line). The solid curve corresponds to the calculation without surface tension, and tends to a straight line (power law) as the flow evolves $\left(X_{m} \rightarrow 0\right)$, indicating a self-similar behavior for $X_{m} \leqslant 0.04$. The measurements are in excellent agreement with the corresponding numerical curve, but they depart from the $\gamma=0$ solution near the front. As expected, the larger $x_{0}$, the nearer to the front this departure begins; however, even for the larg- 


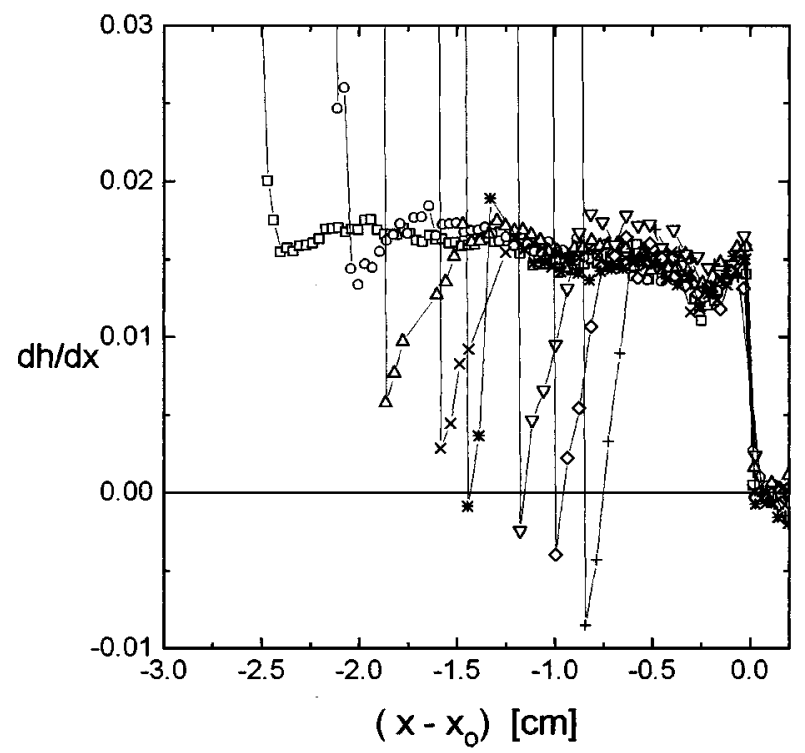

FIG. 9. Sequence of $\partial h / \partial x$ profiles for the cases $x_{0}=75.5 \mathrm{~cm}$ and $\alpha=0.0126$. The dip preceding the corner layer is clearly visible.

est $x_{0}$ the surface tension prevents the development of the self-similar flow predicted by the $\gamma=0$ solution. Clearly, we find that (for a given $X_{m}$ ) the cases with smaller $x_{0}$ produce smaller values of $S_{m}$ and broader $\partial h / \partial x$ peaks, as must be expected since capillarity tends to reduce the local curvature of the profile.

\section{E. Depression preceding the corner layer in the slope profiles}

Near the end of the waiting stage, the $\partial h / \partial x$ profiles develop a peculiar small depression (dip) preceding the corner layer (see Fig. 9), almost imperceptible in the $h(x)$ profiles. This unexpected feature appears for large $S_{m}$, but still into the range of our slice light technique. In Fig. 8 we encircle the points corresponding to profiles where the dip is present. Note that surface tension strongly affects all these profiles, which suggests that capillarity plays a role on the formation of the dip. This guess is enforced by a theoretical model (with dominant capillary effects) reported by Tanner [32], where analogous depressions appear ahead a front advancing over a thin fluid layer. We leave a more detailed study of this interesting phenomenon for a future work.

\section{SUMMARY AND CONCLUSIONS}

The viscous flows produced by a wedgelike initial profile ( $\alpha \leqslant 0.12, x_{0} \geqslant 100 a$ ) display the main features of the waiting front phenomenology and can be described with a reasonable approximation by the solutions of Eq. (1). Surface tension effects are less important for long wedges, but cannot be neglected in experiments; they may be ignored only for natural phenomena occurring on a much larger scale than the capillary length. The flow has a long stage with a motionless front during which the profile changes gradually. The most noteworthy feature of this stage is the evolution of a thin transition region (corner layer), where the slope of the liquid free surface varies strongly, propagating toward the front. The existence of this feature has been predicted theoretically, but its properties have not been investigated in detail previously. The corner layer separates two regions clearly: one ahead, almost unperturbed, and another behind, strongly modified with respect to the initial condition. The front starts to move precisely when it is overtaken by the corner layer. As the corner layer approaches the front, its evolution tends to a self-similar regime $\left(X_{m} \leqslant 0.04\right)$ in which $X_{m}$ varies according to a power law of $T$, independently of $\nu, \alpha$, and $x_{0}$. The self-similar solution of the first kind, which describes the spreading of the same liquid volume delivered at $t=0$ and $x=0$, provides very good estimates of some magnitudes. First, $t_{w}$ is close to the time at which, according to this solution [Eq. (3)], the front arrives at $x_{0}$; second, both the velocities of the corner layer (before $t_{w}$ ) and the moving front (after $t_{w}$ ) are well given by the front velocity corresponding to this solution (except near $x_{0}$ ). The experimental waiting-time values are always within the bounds provided by Aronson and co-workers [14,26] and Vazquez [27], i.e., $0.3 \leqslant t_{w} / t^{*} \leqslant \infty$ and $0.52 \leqslant t_{w} / t^{*} \leqslant 29.87$, respectively.

The results not related to the mathematical properties of Eq. (1) can be summarized as follows:

(a) The waiting time shows an unexpected slight dependence on the initial aspect ratio $\alpha$ (Fig. 3). This effect has a global character and is related to the experimental boundary condition at the rear wall, which cannot be adequately reproduced within the lubrication theory approximation used in Eq. (1). For a value of $\alpha$ not too large $(<0.12)$, this effect can be accounted by using an effective length in the characteristic time $t^{*}$, slightly less than the actual length of the wedge.

(b) When the corner layer approaches the front $(T \rightarrow 0)$, the shape of the $\partial h / \partial x$ profile near $S_{m}$ and the evolution of the maximum slope (Fig. 8) differ from the solution without surface tension; this indicates that the flow in the peak region departs from self-similarity. Associated with this deviation, a small dip of the $\partial h / \partial x$ distribution appears ahead of the peak (Fig. 9). These departures are sensitive to $x_{0}$ but not to $\alpha$. Our numerical simulations show that these are local effects mainly due to capillarity, that prevents the complete development of the self-similar regime in the corner layer region. In our experiments, the influence of surface tension on the corner layer precedes the two-dimensional effects associated with the breakdown of the lubrication hypothesis (that occurs due to $\partial h / \partial x \rightarrow \infty$ when $x_{m} \rightarrow x_{0}$ ).

(c) For a value of $t$ larger but close to $t_{w}$, we find that the front moves with an almost constant velocity (i.e., $\delta_{b} \approx 1$ ) according to the predictions of Aronson, Caffarelli, and Vazquez [26] and Vazquez [27] for this case. The initial front velocity scales as $\left(x_{0} / t^{*}\right) f(\mathrm{Ca})$ (Fig. 6), where $f$ is an unknown function which tends to a constant for $\mathrm{Ca} \rightarrow \infty$. This shows that capillarity is also important after the front starts to move if $\mathrm{Ca} \approx 1$ as in experiments.

This work shows that the waiting-time solutions of Eq. (1) better describe the evolution of very long and shallow liquid wedges. The main deviations from pure nonlinear diffusion are due to capillarity, which affects the flow near the corner layer close to the end of the waiting stage, and near the front after it begins to move. These interesting perturbing effects may deserve more specific studies. In our experiments these departures are significant, but do not obscure the basic fea- 
tures of the waiting-time phenomenon, whose study is the main purpose of this paper.

The development and evolution of the corner layer provide a fine and interesting demonstration of the interplay between nonlinear propagation, which tends to reduce the width of the corner layer and to increase the $\partial h / \partial x$ peak to produce a corner shock, and (nonlinear) diffusion, which opposes this tendency, smoothing the $\partial h / \partial x$ profile and broadening the layer. As the flow evolves and the corner layer approaches the front (where the thickness of the current is small) the nonlinear propagation effect prevails. The numerical solutions of Eq. (1) (pure nonlinear diffusion) suggest that the corner layer becomes a corner shock at $t=t_{w}$ when it overtakes the front. Even if in the experiments the corner layer profile is affected by capillarity and two-dimensional effects near the end of the waiting stage, this evolution is clearly displayed as a trend.

\section{ACKNOWLEDGMENTS}

This work was supported in part by CONICET and CIC. J.G. wishes to acknowledge support from the EC (Grant No. CIT ${ }^{*}$ CT91-0944) and the University of Buenos Aires (Project No. EX245).
[1] R. Seshadry and T. Y. Na, Group Invariance in Engineering Boundary Value Problems (Springer-Verlag, New York, 1985).

[2] L. A. Peletier, in Applications of Nonlinear Analysis in the Physical Sciences, edited by H. Ammam and N. Bazley (Pitman, Boston, 1981), p. 229.

[3] J. Bear, Dynamics of Fluids in Porous Media (American Elsevier, London, 1982).

[4] A. A. Lacey, J. R. Ockendon, and A. B. Tayler, J. Appl. Math. 42, 1252 (1982).

[5] J. Buckmaster, J. Fluid Mech. 81, 735 (1977).

[6] H. E. Huppert, J. Fluid Mech. 121, 43 (1982).

[7] J. R. Lister and R. C. Kerr, J. Fluid Mech. 203, 215 (1989).

[8] D. P. Hoult, Annu. Rev. Fluid 4, 341 (1972).

[9] J. E. Simpson, Annu. Rev. Fluid Mech. 14, 213 (1982).

[10] H. E. Huppert, J. Fluid Mech. 173, 557 (1986).

[11] R. C. Kerr and J. R. Lister, Earth Planet. Sci. Lett. 85, 241 (1987).

[12] B. M. Marino, L. Thomas, J. Diez, and R. Gratton, J. Colloid. Interf. Sci. 177, 14 (1996).

[13] W. L. Kath and D. S. Cohen, Stud. Appl. Math. 67, 79 (1982).

[14] D. G. Aronson, L. A. Caffarelli, and S. Kamin, SIAM J. Anal. 14, 639 (1983).

[15] R. E. Pattle, Q. J. Mech. Appl. Math. 12, 407 (1959).

[16] G. I. Barenblatt, Similarity, Self-Similarity, and Intermediate Asymptotics (Consultants Bureau, New York, 1979).
[17] J. A. Diez, R. Gratton, and J. Gratton, Phys. Fluids A 4, 1148 (1992).

[18] J. Gratton, Fund Cosmic Phys. 15, 1 (1991).

[19] Ya. B. Zel'dovich and Yu. P. Raizer, Physics of Shock Waves and High Temperature Hydrodynamics Phenomena (Academic, New York, 1967).

[20] D. G. Aronson, SIAM J. Appl. Math. 19, 299 (1970).

[21] B. F. Knerr, Trans. Am. Math. Soc. 234, 381 (1977).

[22] S. Kamin, in Free Boundary Problems, edited by E. Magenes (Tecnoprint, Rome, 1980).

[23] N. F. Smyth and J. M. Hill, IMAJ J. Appl. Math. 40, 73 (1988).

[24] J. Gratton and C. Vigo, An. AFA (to be published).

[25] J. Gratton, E. Rossello, and J. A. Diez, An. Acad. Nac. Cien. Exactas Fis. Nat. Buenos Aires 1992, 51.

[26] D. G. Aronson, L. A. Caffarelli, and J. L. Vazquez, Commun. Pure Appl. Math. 38, 375 (1985).

[27] J. L. Vazquez, Trans. Am. Math. Soc. 285, 717 (1984).

[28] J. A. Diez, R. Gratton, L. Thomas, and B. Marino, Phys. Fluids 6, 24 (1994).

[29] K. J. Ruschak, Rev. Fluid Mech. 17, 65 (1985).

[30] H. A. Barnes, J. F. Fulton, and K. Walters, An Introduction to Rheology (Elsevier, New York, 1989).

[31] R. R. Rahalker et al., Proc. R. Soc. London Ser. A 394, 207 (1984).

[32] L. H. Tanner, J. Phys. D 12, 1473 (1979).

[33] J. D. Chen, J. Colloid. Interf. Sci. 122, 60 (1987). 